\title{
Prevalence and antimicrobial susceptibility pattern of Staphylococcus Aureus isolated from clinical specimens at the Mater Hospital Nairobi, Kenya.
}

Christina Okello (D okellochristina@yahoo.com )

Mater Hospital https://orcid.org/0000-0002-0693-4715

Loice Achieng

University of Nairobi

Enoch Omonge

University of Nairobi

Frederick Otieno

University of Nairobi

Davies Otieno

Nairobi west hospital

Christina Mwachari

Mater Hospital

\section{Research Article}

Keywords: MRSA - Methicillin resistant Staphylococcus aureus, PSSA- Penicillin susceptible Staphylococcus aureus

Posted Date: December 2nd, 2020

DOI: https://doi.org/10.21203/rs.2.23946/v3

License: (9) This work is licensed under a Creative Commons Attribution 4.0 International License.

Read Full License 


\section{Abstract}

Staphylococcus aureus (S. aureus) is a leading cause of hospital and community acquired infections globally. Methicillin-resistant Staphylococcus aureus (MRSA) prevalence has been reported to be high in various settings and is associated with increased morbidity, mortality and risk of nosocomial outbreaks. Surveillance of prevalence and antibiotic susceptibility patterns is important to ensure appropriate antibiotic prescription. The objective of our study was to establish the prevalence of $S$. aureus, to identify patterns of susceptibility to commonly used antibiotics and quantify contemporary penicillin resistance among $S$. aureus. A retrospective study was conducted at the Mater Misericordiae Hospital, Nairobi. The study involved a review of non-duplicate records of specimens analyzed between January 2014 and December 2018.A specimen was categorized as Penicillin susceptible (PSSA) if susceptible to Penicillin and Oxacillin, MSSA if resistant to Penicillin but susceptible to Oxacillin, and MRSA if resistant to Oxacillin. We present proportions of $S$. aureus that was PSSA, MSSA and MRSA. Multivariate logistic regression was used to determine the association between the presence of $S$. aureus isolates and the source of the clinical specimen (in vs. outpatient), age and gender. A total of 659 specimens of $S$. aureus were analyzed in the 5-year period. PSSA was the most prevalent organism seen (60.85\%) while MRSA was the least prevalent $(0.61 \%)$. Most $S$. aureus was isolated in pus from wound swabs-644 (73. 3\%). A significant increase in susceptibility of $S$. aureus to Penicillin and Amoxicillin-clavulanic acid was observed during the study period however, sensitivity to Amoxicillin declined. This study demonstrated a high prevalence of Penicillin Sensitive $S$. aureus and a low prevalence of MRSA.

\section{Background}

Staphylococcus aureus (S.aureus) is among the most common and devastating human bacterial pathogens, estimated to cause about $20 \%-30 \%$ of bloodstream and surgical site infections globally, as well as up to half of bone and joint infections (1). Antibiotic resistance has emerged as a major problem in the treatment of infections by $S$. aureus. Methicillin resistant $S$. aureus (MRSA) was first reported in the early 1960s, after the introduction of Methicillin following the acquisition of the mecA-containing Staphylococcal cassette chromosome mec(SCCmec) (2). MRSA strains are not only resistant to nearly all beta-lactams, but many have developed resistance to multiple other classes of antimicrobials (3). The epidemiology of MRSA infections has been marked by sequential "waves" of epidemic clones spreading across geographic regions, nations, and continents. As MRSA has become endemic, the use of Vancomycin for therapy of invasive MRSA infections has increased, along with concerns about development of Vancomycin resistance (4). The prevalence of MRSA blood infections in the United States and Europe has dropped in recent years (5). Similarly, MRSA infection rate in Asia is also declining (6). In contrast, recent data suggests that the prevalence of MRSA in most African countries is rising, although the rate is estimated to be below $50 \%$. (7). The changing trajectory of MRSA infection in developed countries has been attributed to implementation of control interventions (2). Recent data also suggests that Penicillin susceptibility may be in a period of renaissance (8). 
Data on MRSA prevalence in Africa has been variable and inconsistent due to lack of effective and systematic routine surveillance systems (9).

The objective of our study was to establish the prevalence of $S$. aureus, to identify patterns of susceptibility of $\mathrm{S}$. aureus to commonly used antibiotics and quantify contemporary penicillin resistance among S. aureus in a tertiary center in Nairobi, Kenya. This information is essential in guiding the choice of antibiotic therapy and will serve as a baseline for measuring the impact of interventions.

\section{Methods}

This study was carried out at the microbiological laboratory at the Mater hospital, a tertiary referral and training hospital in Nairobi, Kenya. The hospital serves a large and diverse low and middle income population comprising patients of African and Asian descent and few Caucasians from Nairobi and its environs. The Mater hospital microbiological laboratory is ISO 2012 certified with a full time clinical microbiologist. A total of 9000 culture specimens were analyzed in the laboratory between 2014 and 2018. We reviewed data on all S. aureus cultures isolated during this period.

\section{Data and sample collection}

This was a retrospective study based on electronic laboratory records from the Mater microbiological laboratory. The following demographic information was extracted from the hospital administrative database about each patient- in vs. outpatient status at time of sample collection, age and gender, and antimicrobial susceptibility profiles of $S$. aureus. Data from 2014 to 2018 was retrieved from the hospital medical and laboratory records and for 2018 from Vitek 2 (12) antibiotic susceptibility system imported to a database. (13) Samples collected within the hospital and its satellite clinics are routinely cultured on blood agar and mannitol salt agar and identified to be $S$. aureus using catalase and coagulase tests. Tests are performed in vitro, and the growth response of an isolated organism to a particular drug or drugs are measured. These tests are performed under standardized conditions so that the results are reproducible. The raw data is either in the form of a zone size or MIC.

After incubation, the diameters of the complete growth inhibition zones around each disk are measured. Pre-specified breakpoints are used to interpret the zone sizes and classify them as susceptible(S), intermediate (I), or resistant(R). The most common method employed by the Mater hospital diagnostic laboratory between 2014 and 2018 was a simpler agar disk diffusion test (Kirby-Bauer method).

Clinical Laboratory Standards Institute (CLSI) M100-S19 breakpoints were used for interpretation. Isolates that were "intermediate" by CLSI breakpoints were grouped with resistant isolates for all analyses. Methicillin resistance during this period was determined using Oxacillin. This method used in the Mater laboratory was similar to methods of MRSA identification used elsewhere (14). Isolates from a solution adjusted to $0.5 \mathrm{McF}$ arland standard were spot inoculated unto Mueller Hinton agar supplemented with 6 micrograms $/ \mathrm{mL}$ oxacillin and $4 \%$ sodium chloride. The plates were incubated at 
$35^{\circ} \mathrm{C}$ for 24 hours. The isolates that survived showing more than one colony were considered Methicillin resistant.

Approval for this retrospective study was obtained from the Ethics committee of the Mater hospital. All patient information was de-identified prior to analysis.

\section{Data Analysis}

The data collected was analyzed using Statistical Package for Social Sciences (SPSS) (15). We categorized a specimen a priori, as Penicillin Susceptible Staphylococcus aureus (PSSA) if it was susceptible to Penicillin and Oxacillin, Methicillin Susceptible Staphylococcus aureus (MSSA) if it was resistant to Penicillin and susceptible to Oxacillin. MRSA were resistant to Oxacillin. Multivariate logistic regression was used to determine the association between the presence of $S$. aureus isolates and the source of the clinical specimen (in vs. outpatient), age and gender. A P- value $<0.05$ was considered statistically significant. A new case of $S$. aureus in the same patient was reported if the organism was isolated 21 or more days from the date of the first positive culture.

\section{Results}

\section{Prevalence;}

A total of 659 non-duplicate specimens with $S$. aureus isolates were included in the study. Initially we had 879 specimen collection forms however 220 were excluded due to incomplete data.

PSSA was the most prevalent organism seen in all S. aureus cultures during the study period $(60.85 \%$, $401 / 659)$ while MRSA species were the least prevalent $(0.61 \%, 4 / 659) .38 .54 \%(254 / 659)$ of the isolates were MSSA.

The annual prevalence of MRSA fluctuated between 0-1\% during the five-year study period.

\section{Demographics}

$62.37 \%(411)$ of all specimens were collected from outpatients as compared to $37.63 \%$ ( 248$)$ from inpatients. All MRSA isolates were cultured from inpatient samples.

The average age of all patients with $S$. aureus infections $(\mathrm{N}=659)$ was 29.66 , Females constituted the majority of the study population-503/659, (76.33\%). MRSA was seen more in male patients $(3 / 4,75 \%)$ unlike MSSA (100/254, 39.37\%) and PSSA (52/401,12.97\%).

S. aureus was largely isolated from wound swabs - $(589 / 659,89.38 \%)$. The prevalence of $S$. aureus bacteremia was $10.62 \%(70 / 659)$

Similarly, MRSA reported was isolated in pus. The 2 pus specimens were obtained from an infected surgical wound and a diabetic foot infection. One MRSA was isolated from cerebrospinal fluid. 


\section{Susceptibility patterns}

All organisms isolated were sensitive to Vancomycin, Clindamycin and Linezolid. Oxacillin sensitive organisms accounted for $99.39 \%$ of the study population. (Figure 2)

$(60.85 \%, 401 / 659),(70.71 \%, 466 / 659)$ and $(83.31 \%, 549 / 659)$ of isolates were susceptible to Penicillin, Trimethoprim/sulfamethoxazole and Erythromycin respectively.

Of the organisms isolated, $98.94 \%, 98.03 \%$ and $99.70 \%$ respectively were sensitive to Gentamicin, Ciprofloxacin and Teicoplanin.

As a sub analysis we looked at the trend in penicillin resistance during the study duration. We focused on 3 commonly used penicillins in our setting-Amoxicillin, Amoxicillin-clavulanic acid and Penicillin $\mathrm{G}$. The annual resistance to Penicillin $\mathrm{G}$ and Amoxicillin/clavulanic acid declined over this period, a trend that was statistically significant -Penicillin G (P value $<0.05,95 \% \mathrm{Cl} 4.6376-28.3464)$ Amoxicillin-clavulanic acid ( $\mathrm{P}$ value, $<0.001,95 \% \mathrm{Cl} 37.50-85.83$ ). (Figure 3) However, a significant increase in resistance to Amoxicillin was noted in the same period ( $P$ value $<0.001,95 \% \mathrm{Cl}-50.4$ to -29.44 )

Multivariate logistic regression confirmed no significant association between the probability of Penicillin resistance and site of collection (in vs. outpatient), age or gender. ( $P=0.35,0.98$ and 0.07 respectively.

\section{Trends in mean antibiotic resistance}

In 2014 a S. aureus isolate was on average resistant to 3.02 antibiotics and by 2018 this decreased to 1.8 antibiotics.

The number of MRSA isolated was too few to allow any further exploration of risk factors.

\section{Discussion}

In this retrospective review of a large number of Staphylococcus isolates, we demonstrate a low prevalence of MRSA and high $S$. aureus susceptibility to penicillins. A similar prevalence has been reported in previous studies (11). One study reviewed 731 specimens of $S$. aureus isolated in two private hospitals in Kenya found a low prevalence of MRSA (3.7\%). This could be explained by low community transmission of MRSA (16).

MSSA and PSSA were predominantly isolated in outpatients unlike MRSA which was more in admitted patients. This has similarly been observed in Japan where MRSA was more prevalent in admitted patients. (17) Even though this study was not designed to identify risk factors for MRSA acquisition, our site is not unique as it shares risk factors commonly associated with nosocomial acquisition of MRSA such as broad-spectrum antimicrobial therapy and admissions to intensive care units (18).

Majority of $S$. aureus isolates were from specimens cultured from patients between the ages of 0-19. In a study carried out in Australia (19), MRSA infections were found to occur more often in younger patients 
(under 40). This transmission could be explained by the fact younger people may have close skin-to-skin contact more often during participation in sports or when working.

Most $S$. aureus isolates were from female patients. This difference could have arisen from the fact that female patients constituted the majority of the study population $(76.33 \%)$ and this could further be explained by the differences in health seeking behavior across different genders (20).

MSSA, PSSA and MRSA were all predominantly isolated from pus. This finding is similar to what was found by Dilnessa et al in Ethiopia (18). MRSA was also isolated from a tracheal aspirate specimen and cerebrospinal fluid. The three clinical situations that predispose to the development of $S$ aureus meningitis are neurosurgical intervention, contiguous infection and $S$ aureus bacteremia. Until recently, most cases have been caused by Methicillin-susceptible $S$ aureus strains. There are now an increasing number of reports of severe infections attributed to methicillin-resistant strains of $S$ aureus (22).

Methicillin Resistant $S$. aureus cultured in our setting was sensitive to Linezolid, Vancomycin, Tigecycline, Rifampicin, Fusidic acid, Teicoplanin and Tetracycline. These antibiotics have proved effective for treatment of MRSA infections in other parts of the world (23), (24). On the contrary, all the MRSA were resistant to Clindamycin which is considered an option in MRSA treatment in some parts of the United States of America(USA).

In our study half of MRSA isolates were susceptible to Trimethoprim-sulfamethoxazole. (26) Reported resistance of MRSA to Trimethoprim-sulfamethoxazole has been found to vary worldwide, in general being low in the industrialized world and higher in developing countries.

Notably, in a sub analysis we found that resistance of $S$. aureus to Amoxicillin-Clavulanic acid and Penicillin G declined gradually over the past five years however, resistance to Amoxicillin rose. This could be explained by the overuse of Amoxicillin which is more readily available and cheaper than amoxicillinclavulanic acid and a decline in use of Penicillin G over the years. (27) Furthermore, recent publications have reported a rise of Penicillin-Sensitive Staphylococcus aureus. This trend has now been observed on several continents (8). In the United states, this increase in the incidence of PSSA infections has also been mirrored by a decrease in Methicillin-resistant $S$. aureus infection rates (25).

Overall antibiotic resistance in $S$. aureus in this site has declined in the past 5 years. One potential explanation for the trend of increasing $S$. aureus antibiotic susceptibility is a shift in antibiotic pressures. The decline in the use of narrow-spectrum beta-lactams such as Oxacillin and Penicillin G since 2000 and the inpatient use of first-generation cephalosporins since 2006 may select against hospital acquired MRSA in favor of PSSA (2).

There are several limitations to our study. First, the small number of MRSA preclude further analysis of risk factors and treatment options. Conclusions about MRSA as pertains to the choice of antibiotics for treatment of MRSA infections, in this setting must be interpreted carefully. Secondly, the testing for 
inducible beta-lactamase production was not performed. In addition, in the absence of genotyping of specimens, phenotypic MRSA identification methods may not reflect an accurate picture.

\section{Conclusion}

The prevalence of MRSA was low. There were no strains that were resistant to Vancomycin, the drug of choice for MRSA. There has been a decline in resistance of $S$. aureus to Penicillin and Amoxicillinclavulanic acid in the past five years.

\section{Abbreviations}

SCC mec- Staphylococcal cassette chromosome mec

MIC- Minimum inhibitory concentration

SSI- Skin and soft tissue infection

MSSA- Methicillin-susceptible Staphylococcus aureus

MRSA-Methicillin-resistant Staphylococcus aureus

CLSI-Clinical and Laboratory Standard Institute

VRSA-Vancomycin Resistant Staphylococcus aureus

OXA-Oxacillin

PEN-Penicillin

VAN-Vancomycin

SXT-Trimethoprim -sulfamethoxazole

GEN-Gentamicin

CLI-Clindamycin

CIP-Ciprofloxacin

LZD-Linezolid

ERY-Erythromycin

TEC-Teicoplanin

FA-Fusidic Acid 
TET-Tetracycline

MXF-Moxifloxacin

RIF-Rifampicin

\section{Declarations}

\section{Authors' contributions}

CO participated in the study design, literature search, data collection, data analysis. CO and LAO wrote the initial manuscript. LAO, EO, FCF, DO and CM participated in the interpretation of data, supervision of the microbiological diagnosis and served as scientific advisors. All authors read and approved the final manuscript.

\section{Funding}

There was no funding for this study

\section{Acknowledgements}

We gratefully acknowledge the assistance of Allan Ng'ang'a, David Shiraku, Gerald Mwangi, and James Kanyingi for their support during the study. A preprint of this work was posted on Research square.

\section{Competing interests}

The authors declare that they have no competing interests

\section{Availability of data and materials}

The data and information supporting the conclusions of this article are available from the corresponding author on reasonable request.

\section{Consent for publication}

Not applicable.

\section{Ethics approval and consent to participate}

The study protocol and procedure were approved by the ethics committee of the Mater hospital dated $9^{\text {th }}$ November 2017. Our study does not include new clinical procedures, nor was it based on laboratory investigations, only on microbiological surveillance data. Our study did not involve any animals.

\section{References}


1. Diekema DJ, Pfaller MA, Shortridge D, Zervos M, Jones RN. Twenty-year trends in antimicrobial susceptibilities among Staphylococcus aureus from the SENTRY Antimicrobial Surveillance Program. Open Forum Infect Dis [Internet]. 2019 Mar 15 [cited 2020 Aug 25];6(Supplement_1):S4753. Available from: https://academic.oup.com/ofid/article/6/Supplement_1/S47/5381623

2. Kanjilal S, Abdul Sater MR, Thayer M, Lagoudas GK, Kim S, Blainey PC, et al. Trends in antibiotic susceptibility in staphylococcus aureus in Boston, Massachusetts, from 2000 to 2014. J Clin Microbiol [Internet]. 2018 Jan 1 [cited 2020 Aug 25];56(1). Available from: https://pubmed.ncbi.nlm.nih.gov/29093105/

3. Diekema DJ, Pfaller MA, Turnidge J, Verhoef J, Bell J, Fluit AC, et al. Genetic relatedness of multidrugresistant, methicillin (oxacillin)-resistant Staphylococcus aureus bloodstream isolates from SENTRY antimicrobial resistance surveillance centers worldwide, 1998. Microb Drug Resist [Internet]. 2000 [cited 2020 Aug 29];6(3):213-21. Available from: https://pubmed.ncbi.nlm.nih.gov/11144421/

4. Limbago BM, Kallen AJ, Zhu W, Eggers P, McDougal LK, Albrecht VS. Report of the 13th vancomycinresistant Staphylococcus aureus isolate from the United States. J Clin Microbiol [Internet]. 2014 Mar [cited 2020 Aug 27];52(3):998-1002. Available from: /pmc/articles/PMC3957794/?report=abstract

5. Sutter DE, Milburn E, Chukwuma U, Dzialowy N, Maranich AM, Hospenthal DR. Changing Susceptibility of Staphylococcus aureus in a US Pediatric Population. Pediatrics [Internet]. 2016 Apr 1 [cited 2020 Aug 25];137(4). Available from: www.aappublications.org/news

6. Abubakar U, Sulaiman SAS. Prevalence, trend and antimicrobial susceptibility of Methicillin Resistant Staphylococcus aureus in Nigeria: a systematic review. Vol. 11, Journal of Infection and Public Health. Elsevier Ltd; 2018. p. 763-70.

7. Falagas ME, Karageorgopoulos DE, Leptidis J, Korbila IP. MRSA in Africa: Filling the Global Map of Antimicrobial Resistance. Becker K, editor. PLoS One [Internet]. 2013 Jul 29 [cited 2020 Aug 27];8(7):e68024. Available from: https://dx.plos.org/10.1371/journal.pone.0068024

8. Cheng MP, René P, Cheng AP, Lee TC. Back to the Future: Penicillin-Susceptible Staphylococcus aureus. Am J Med [Internet]. 2016 Dec 1 [cited 2020 Aug 29];129(12):1331-3. Available from: http://dx.doi.org/10.1016/j.amjmed.2016.01.048

9. Wangai FK, Masika MM, Maritim MC, Seaton RA. Methicillin-resistant Staphylococcus aureus (MRSA) in East Africa: Red alert or red herring? BMC Infect Dis [Internet]. 2019 Jul 9 [cited 2020 Aug 29];19(1):596. Available from: https://bmcinfectdis.biomedcentral.com/articles/10.1186/s12879019-4245-3

10. Gitau W, Masika M, Musyoki M, Museve B, Mutwiri T. Antimicrobial susceptibility pattern of Staphylococcus aureus isolates from clinical specimens at Kenyatta National Hospital. BMC Res Notes [Internet]. 2018 Apr 3 [cited 2020 Aug 29];11(1):226. Available from: https://bmcresnotes.biomedcentral.com/articles/10.1186/s13104-018-3337-2

11. Omuse G, Kabera B, Revathi G. Low prevalence of methicillin resistant as determined by an automated identification system in two private hospitals in Nairobi, Kenya: A cross sectional study. 
BMC Infect Dis [Internet]. 2015 Feb 6 [cited 2020 Aug 29];14(1). Available from: https://pubmed.ncbi.nlm.nih.gov/25495139/

12. bioMérieux. Vitek 2, bioMérieux. 2018.

13. LIFELINE. LIFELINE DATA MANAGEMENT. 2014.

14. Udobi CE, Obajuluwa AF, Onaolapo JA. Prevalence and antibiotic resistance pattern of methicillinresistant staphylococcus aureus from an orthopaedic hospital in nigeria. Biomed Res Int. 2013;2013.

15. IBM-SPSS. IBM SPSS statistics for Windows. Armonk,N.Y: IBM Corp.; 2018.

16. Aiken AM, Mutuku IM, Sabat AJ, Akkerboom V, Mwangi J, Scott JAG, et al. Carriage of Staphylococcus aureus in Thika Level 5 Hospital, Kenya: A cross-sectional study. Antimicrob Resist Infect Control [Internet]. 2014 Jul 15 [cited 2020 Oct 9];3(1):22. Available from: https://aricjournal.biomedcentral.com/articles/10.1186/2047-2994-3-22

17. Nishijima S, Sugimachi T, Higashida T, Asada Y, Okuda K, Murata K. An epidemiological study of methicillin-resistant Staphylococcus aureus (MRSA) isolated from medical staff, inpatients, and hospital environment in one ward at our hospital. J Dermatol [Internet]. 1992 [cited 2020 Oct 9];19(6):356-61. Available from: https://pubmed.ncbi.nlm.nih.gov/1401489/

18. Dilnessa T, Bitew A. Prevalence and antimicrobial susceptibility pattern of methicillin resistant Staphylococcus aureus isolated from clinical samples at Yekatit 12 Hospital Medical College, Addis Ababa, Ethiopia. BMC Infect Dis [Internet]. 2016 Aug 9 [cited 2020 Oct 9];16(1):398. Available from: http://bmcinfectdis.biomedcentral.com/articles/10.1186/s12879-016-1742-5

19. Agostino JW, Ferguson JK, Eastwood K, Kirk MD. The increasing importance of community-acquired methicillin-resistant Staphylococcus aureus infections. Med J Aust [Internet]. 2017 Nov 30 [cited 2020 Oct 9];207(9):388-93. Available from:

https://onlinelibrary.wiley.com/doi/abs/10.5694/mja17.00089

20. Thompson AE, Anisimowicz Y, Miedema B, Hogg W, Wodchis WP, Aubrey-Bassler K. The influence of gender and other patient characteristics on health care-seeking behaviour: A QUALICOPC study. BMC Fam Pract [Internet]. 2016 [cited 2020 Oct 9];17(1). Available from: /pmc/articles/PMC4815064/? report=abstract

21. Humphreys H, Fitzpatick F, Harvey BJ. Gender differences in rates of carriage and bloodstream infection caused by methicillin-resistant staphylococcus aureus: Are they real, do they matter and why? Clin Infect Dis [Internet]. 2015 Dec 1 [cited 2020 Oct 9];61(11):1708-14. Available from: https://academic.oup.com/cid/article/61/11/1708/333596

22. Pereira NMD, Shah I, Ohri A, Shah F. Methicillin resistant staphylococcus aureus meningitis. Oxford Med Case Reports [Internet]. 2015 [cited 2020 Oct 9];2015(11):364-6. Available from: /pmc/articles/PMC4652058/?report=abstract

23. Liu C, Bayer A, Cosgrove SE, Daum RS, Fridkin SK, Gorwitz RJ, et al. Clinical practice guidelines by the Infectious Diseases Society of America for the treatment of methicillin-resistant Staphylococcus aureus infections in adults and children. Clin Infect Dis [Internet]. 2011 Feb 1 [cited 2020 Oct 9];52(3):18-55. Available from: https://academic.oup.com/cid/article/52/3/e18/306145 
24. Khalili H, Dashti-Khavidaki S, Khaleghi S, Maleki Z, Rasoolinejad M. Evaluation of Tigecycline Activity Against Methicillin-Resistant Staphylococcus aureus Isolated from Biological Samples. Iran J Pharm Res IJPR [Internet]. 2010 [cited 2020 Oct 15];9(1):61-5. Available from: http://www.ncbi.nlm.nih.gov/pubmed/24363708

25. Butler-Laporte G, Lee TC, Cheng MP. Increasing rates of penicillin sensitivity in staphylococcus aureus [Internet]. Vol. 62, Antimicrobial Agents and Chemotherapy. American Society for Microbiology; 2018 [cited 2020 Oct 9]. Available from: https://doi.org/10.1016/j.jinf.2016

26. Pappa G, Athanasoulia AP, Matthaiou DK, Falagas ME. Trimethoprim-sulfamethoxazole for methicillin-resistant staphylococcus aureus: a forgotten alternative? [Internet]. Vol. 21, Journal of Chemotherapy. E.S.I.F.T. srl; 2009 [cited 2020 Oct 9]. p. 115-26. Available from:

https://pubmed.ncbi.nlm.nih.gov/19423463/

27. Ongarora D, Karumbi J, Minnaard W, Abuga K, Okungu V, Kibwage I. Medicine Prices, Availability, and Affordability in Private Health Facilities in Low-Income Settlements in Nairobi County, Kenya. [cited 2020 Nov 11]; Available from: www.mdpi.com/journal/pharmacy.

\section{Figures}

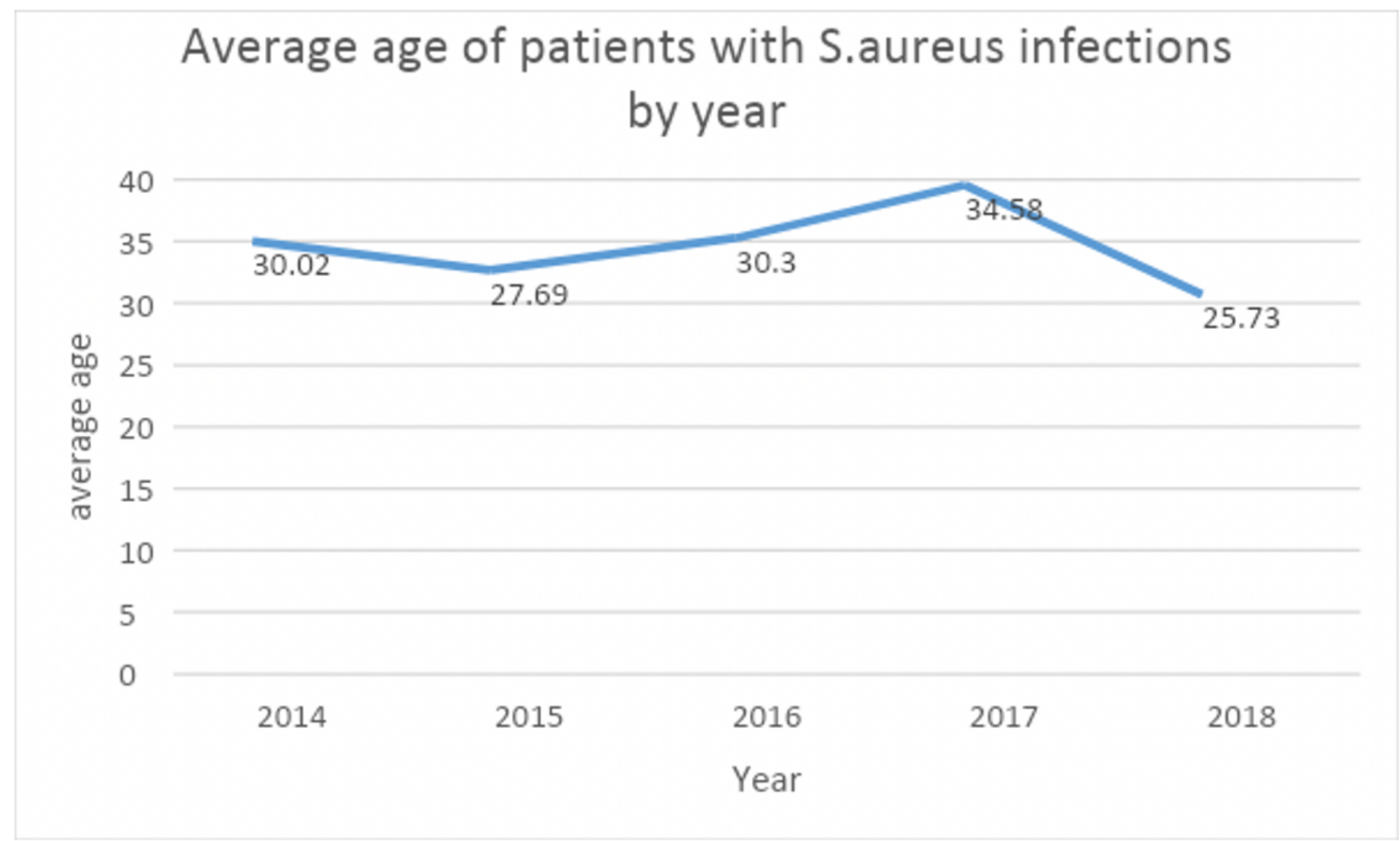

\section{Figure 1}


Graph showing average age of patients with S. aureus infections by year (2014 -2018)

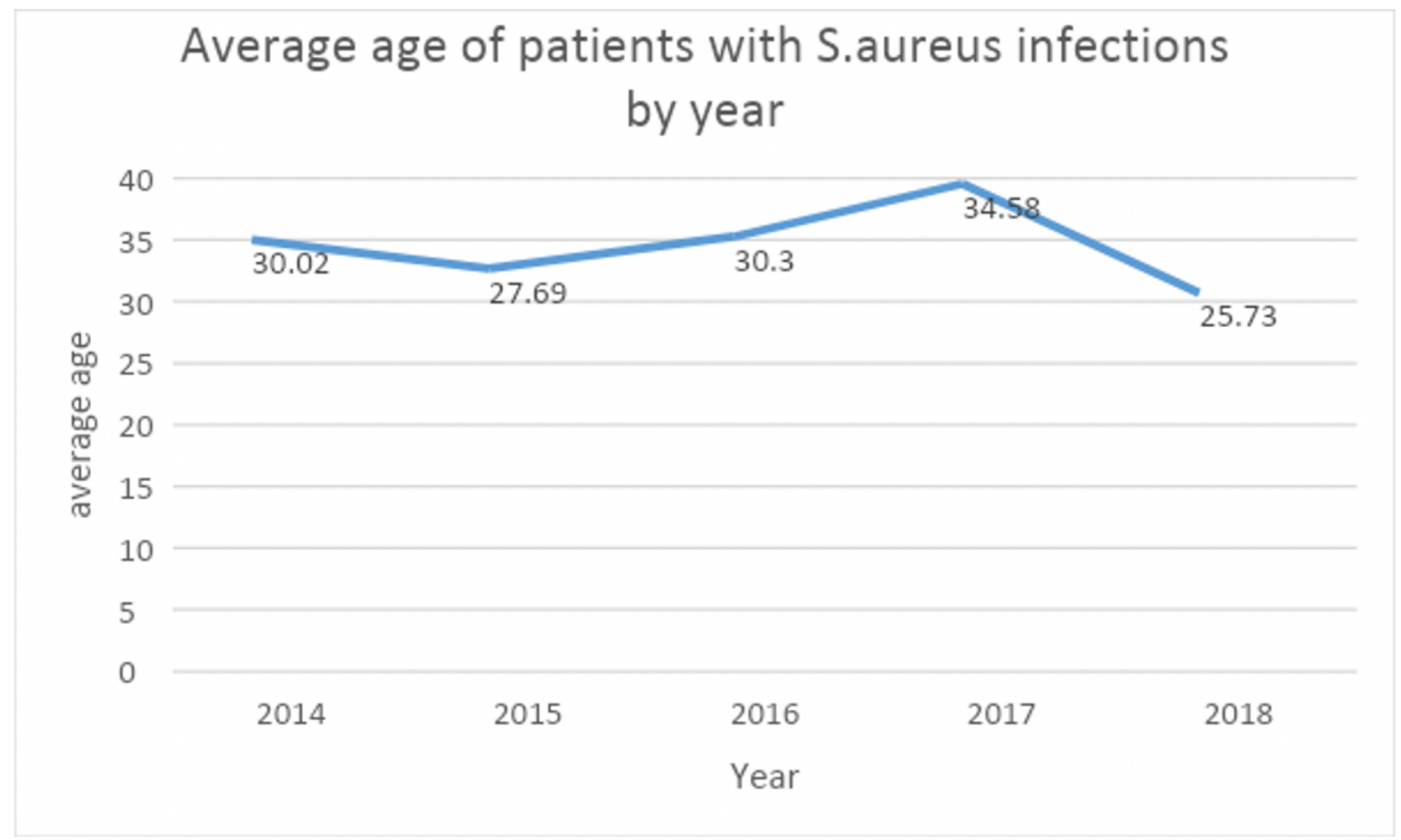

\section{Figure 1}

Graph showing average age of patients with S. aureus infections by year (2014 -2018) 


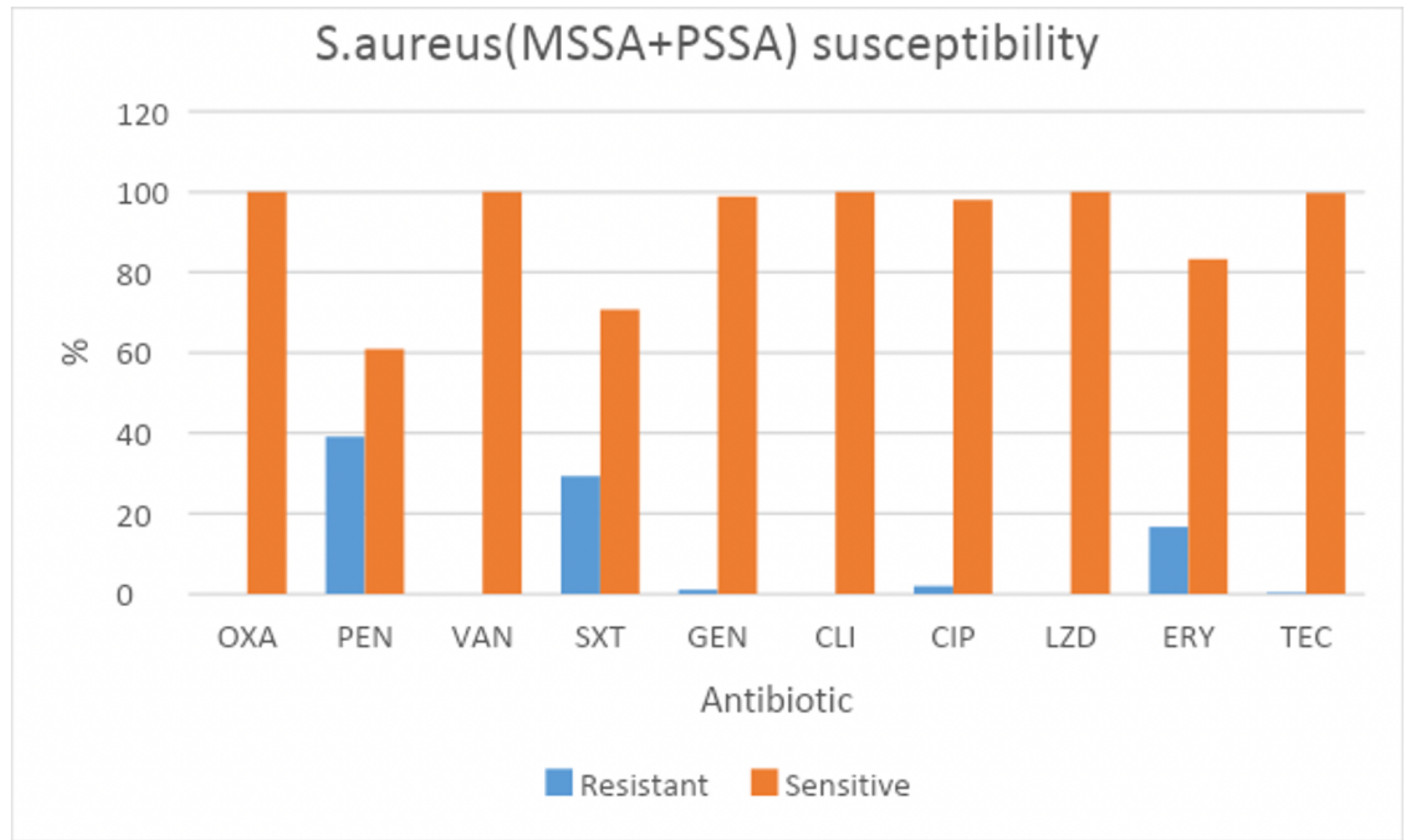

Figure 2

Bar chart displaying percentage of $\mathrm{S}$. aureus susceptible to different antibiotics 


\section{S.aureus(MSSA+PSSA) susceptibility}

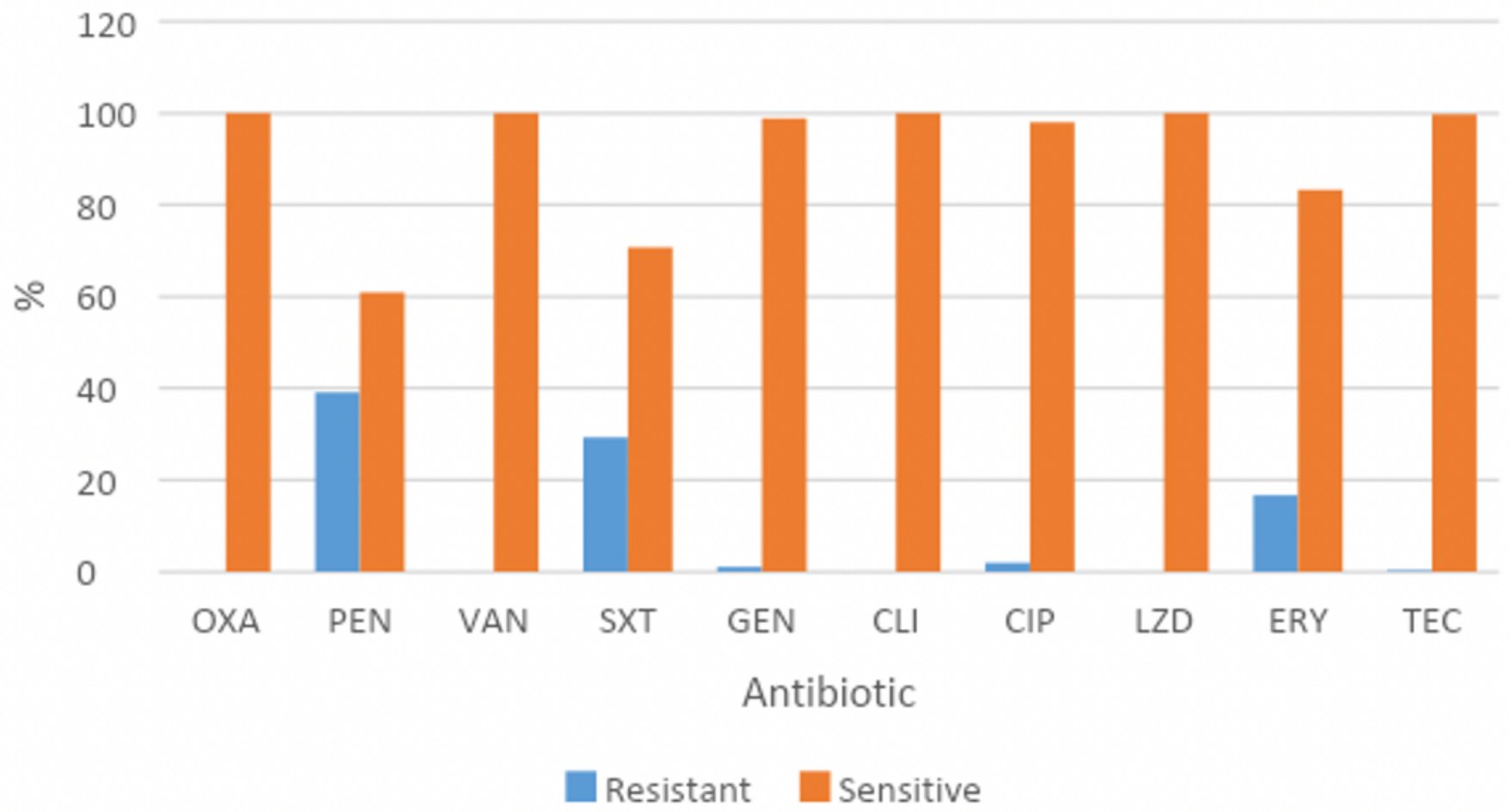

Figure 2

Bar chart displaying percentage of S. aureus susceptible to different antibiotics

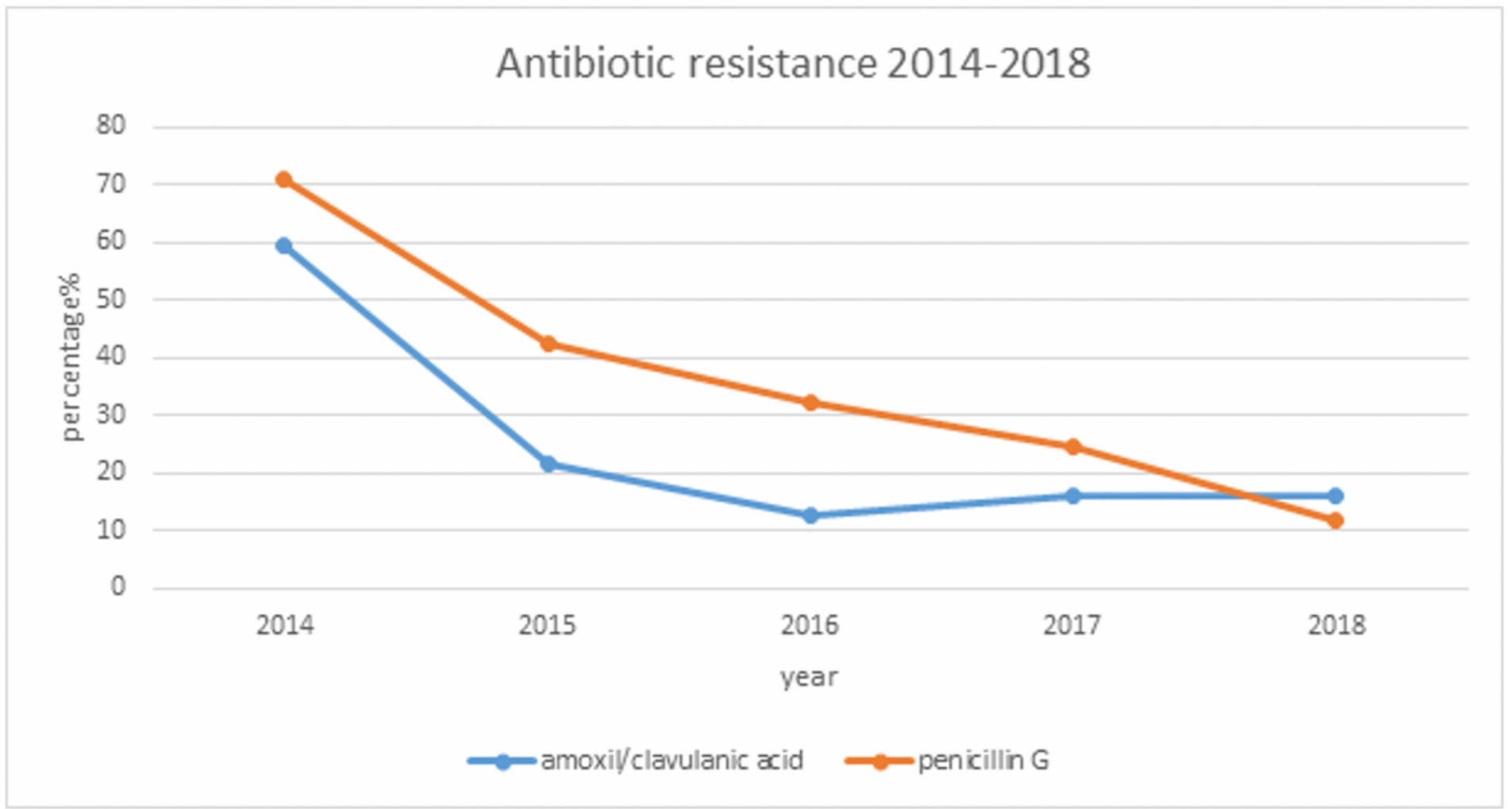




\section{Figure 3}

Graph showing trend of antibiotic resistance by S. aureus to Amoxicillin-Clavulanic acid and Penicillin G between 2014 and 2018

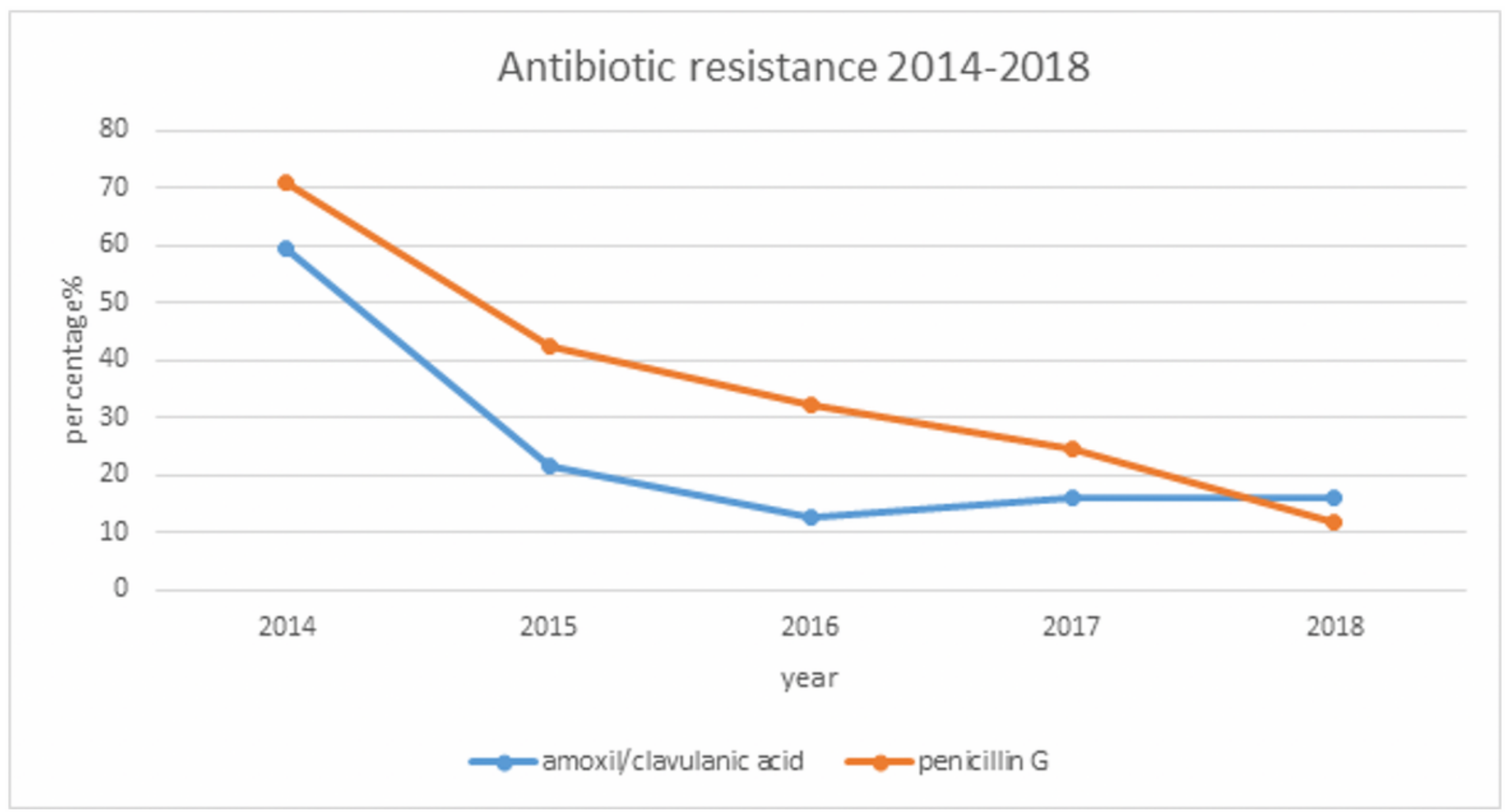

\section{Figure 3}

Graph showing trend of antibiotic resistance by S. aureus to Amoxicillin-Clavulanic acid and Penicillin G between 2014 and 2018 